\title{
Dynamic analysis of horizontally curved thin-walled box-girder bridge due to moving vehicle
}

\author{
K. Nallasivam, Anjan Dutta* and Sudip Talukdar \\ Department of Civil Engineering, Indian Institute of Technology, Guwahati, India
}

Received 9 January 2006

Revised July 2006

\begin{abstract}
The impact on curved box-girder bridges due to vehicle moving across rough bridge deck have been analyzed using bridge-vehicle coupled dynamics. The bridge deck unevenness has been assumed to be a homogeneous random process in space specified by a PSD function. The analysis incorporates the effect of centrifugal forces due to vehicle moving on curved bridge. The curved box-girder bridge has been numerically modeled using computationally efficient thin-walled box-beam finite elements which take into account the torsional warping, distortion and distortional warping, that are important features of thin-walled box girders. Rigid vehicle with longitudinal and transverse input to the wheels giving rise to heave-pitch-roll degrees of freedom has been considered. The theoretical bridge model used in simulation study has been validated by a free vibration experiment using impact excitation. The impact factors for several response parameters such as bending moment, shear force, torsional moment, torsional bi-moment, distortional moment, distortional bi-moment and vertical deflections have been obtained for various bridge-vehicle parameters. Both constant velocity and forward acceleration of the vehicle have been considered to examine impact factor. The results highlighted that the impact factors of a curved box girder bridge corresponding to torsion, distortion and their corresponding bimoments have been observed to be generally very high, while those of the other responses are also relatively higher than that of corresponding straight box girder bridge.
\end{abstract}

\section{Introduction}

The subjects of horizontally curved box-girder bridges have undergone a remarkable development over the past three decades. The primary reasons for the increased popularity of curved box-girder bridges are the increased demand for curved roadway alignment for the smooth dissemination of congested traffic along with the advancement in fabrication and erection technology. However, due to geometric complexities, in addition to experiencing conventional structural action, which are extension, flexure and torsion, cross-section of curved box girders may warp out of the sectional plane and distort in the cross-section. Thus, this type of structure has also inherently created new design problems for engineer.

The problem of vehicle-bridge interaction has been an important topic in the field of civil engineering. In particular, attention is paid to vibrations that might affect the durability of the structure as well as safety and comfort of passengers. Mathematical models have been developed to be able to describe the dynamic behavior of the supporting structure and the vehicle in terms of interaction systems. Bridge vehicle vibration is a complex phenomenon governed by a large number of different parameters such as characteristics of vehicle, characteristics of bridge structure and their mutual influence on each other. In design practice, the dynamic effect caused by the fluctuating force component induced by the moving vehicle is taken into consideration by magnifying the live load

*Corresponding author. Fax: +91 3612690762; E-mail: adutta@iitg.ernet.in. 
effect by a multiplying factor termed as impact factor. The estimation of such impact factor for the curved box girder bridge, while considering all the possible complexities of bridge vehicle interaction has been the motivation of many researchers over the last few years.

The task of the development of appropriate strategy for the analysis of thin-walled box girder bridges were going on for last three decades. Maisel [1], Kermani and Waldron [2] developed a very much generalized method for the analysis of straight box girder under static loading, which accounts for warping torsion and distortion in addition to the more familiar beam actions. However, during the past two decades, the finite-element method of analysis has rapidly become a very popular technique for the solution of complex problems in engineering. A logical alternative for modeling of curved box girder bridges is to combine finite element technique with thin-walled beam theory. Several investigators, notably Zhang and Lyons [3], Razaqpur and Li [4] combined thin-walled beam theory of Vlasov and finite element technique to develop a thin-walled box beam element for elastic analysis of straight and curved cellular bridges. However, the publications dealing with the dynamic response of bridge structure due to moving vehicular load and its impact assessment are scanty. To study the impact factors of horizontally curved steel box-girder bridges, Schelling et al. [5] treated a multi-box girder bridge as a planar grid and the vehicle as two constant forces with no mass. A significant contribution was made by Huang et al. [6], who developed a method for the dynamic analysis of horizontally curved box-girder bridges due to truck loading. Thin-walled beam elements were used, which included both warping torsion and distortion. Major issues concerning vehicle induced vibration in a curved bridge such as the effect of multi-vehicle loadings (side by side) moving over a rough deck at various speeds, appropriate strategy for modeling of vehicle as well as the effect of damping were considered in their study. Huang [7] analyzed the impact on a number of continuous bridges using the same bridge and vehicle model as in Ref. [6]. Impact factor based on different response parameters clearly demonstrated the significance of torsion, distortion in a curved box girder bridge. Yang et al. [8] derived analytical solutions for a horizontally curved beam subjected to moving loads. Centrifugal forces, which are generated due to moving load on a curved path, were also properly accounted for in the evaluation of dynamic response in all the above three referred studies.

Thus, the literature survey showed that a limited number of research studies were conducted on the evaluation of dynamic responses of curved box girder bridges using finite element method. The main focus of the present investigation is to provide elaborate studies on the dynamic response of curved box girder bridges using finite element analysis with emphasis on the evaluation of impact factors corresponding to various stress resultants. The finite element analysis has been performed using an element, which is computationally efficient as well as reasonably representative of thin-walled box girder behaviour. A free vibration experimental study has been conducted on a Perspex sheet model of the curved bridge to validate the natural frequencies of the theoretical bridge model, since natural frequencies are important for understanding the response behaviour. Most of the main parameters that may affect the dynamic response of a curved thin-walled box girder bridge due to moving vehicles have been considered. The impact factors for design as reported in the present investigation have been found to have similar trend as demonstrated by earlier investigators. Moreover, the present study has also demonstrated the influence of forward acceleration of vehicle unlike most of the earlier studies, where constant speeds have been considered and significant differences have been observed due to the influence of vehicular acceleration.

\section{System models}

The dynamic analysis of a bridge due to vehicle-induced vibration is a coupled bridge-vehicle interaction problem with bridge deck surface condition significantly contributing to the overall response of the bridge structure. Thus, appropriate modeling of vehicle, deck surface and the bridge are very much essential for reliable evaluation of dynamic response.

\subsection{Vehicle model}

A bridge is subjected to a variety of vehicular loading. However, simplified studies were conducted with only one point input heave model [9], two point input heave-pitch and heave-roll model [10]. The heave-pitch and heave-roll models incorporate the interaction between two modes at a time and are more complete in comparison 


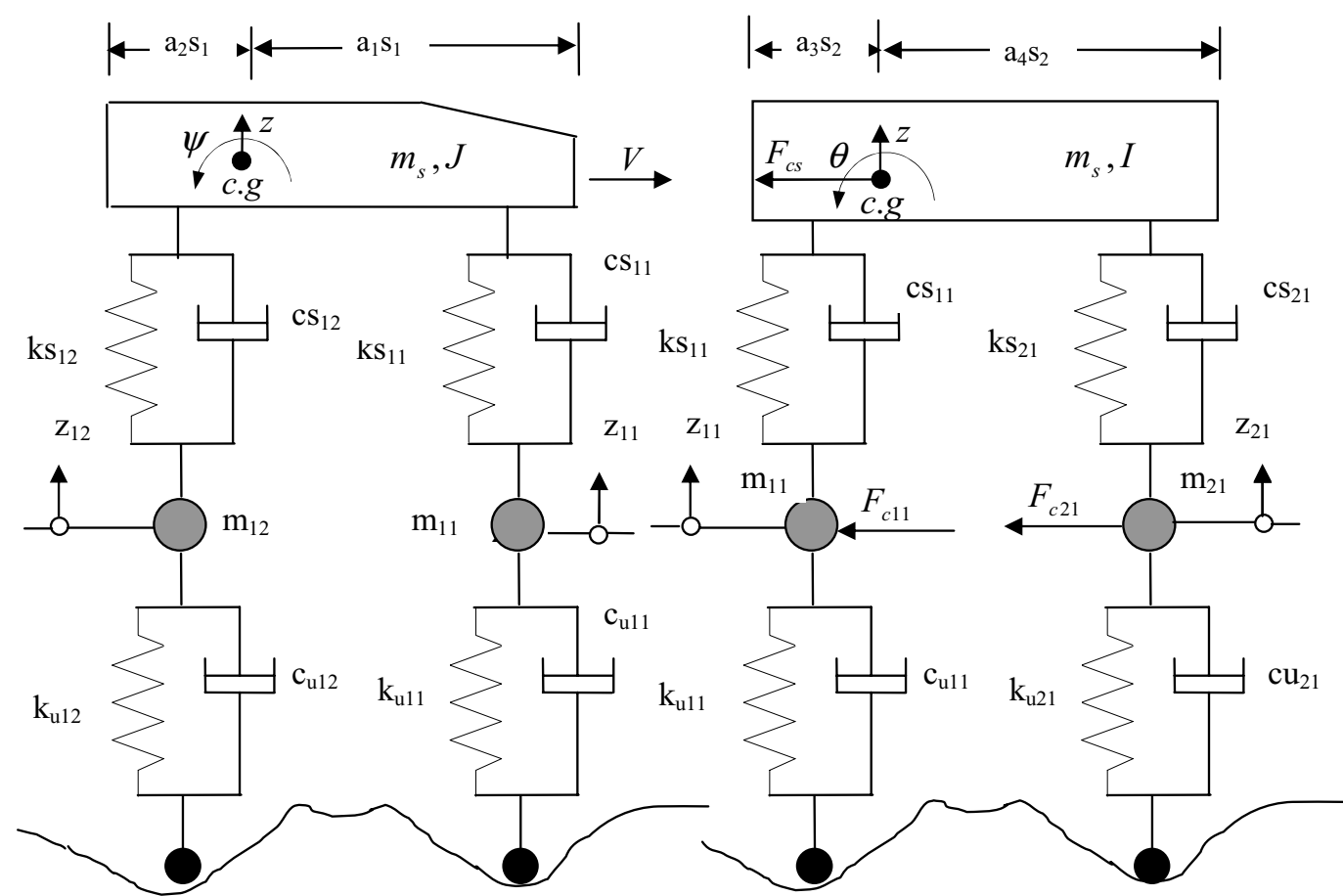

a)

(b)

Fig. 1. 3-D Vehicle model with seven degrees of freedom (a) Side view (b) End view.

to the first method. Further improvements in modeling were achieved by considering a combined heave, pitch and roll dynamics of the vehicle for predicting the coupled oscillation of the system [11]. Huang et al. [12] used the AASHTO HS20-44 truck simulated as a nonlinear tractor and trailer vehicle model with eleven independent degrees of freedom. However, the model of vehicle chosen for the present investigation of the dynamic interaction is seven degrees of freedom, heave-pitch-roll model as shown in Fig. 1. The model is well representative of the prevailing design truck as available in India and surrounding South Asian countries and is fully capable of idealizing all the important motion components of the vehicle and their interaction. The model consists of one sprung mass representing the vehicle and four unsprung masses. The sprung mass has been assigned three degree of freedom, which corresponds to the vertical displacement $z$ (heave motion along vertical axis), rotation about the longitudinal axis $\theta$ (pitch) and rotation about the transverse axis $\psi$ (roll). The unsprung masses are assumed to have only heave motion, a vertical displacement $z_{i p}$. The first suffix $i$ denotes wheel position -1 for the right and -2 for the left. The second suffix $p$ gives the axle sequence, starting with the front axle.

The total seven independent degrees of freedom of the vehicle model can be written as

$$
[D]=\left[z, \psi, \theta, z_{i p}\right] i=1,2 ; p=1,2 \text {; }
$$

The centrifugal force $\left(F_{c}\right)$ of each mass has been assumed to act horizontally at its centroid.

\subsection{Bridge deck roughness}

The bridge deck unevenness causes moving vehicles to pick up dynamic excitation, which in turn imposes oscillatory loads on the deck. The analyst needs to choose an appropriate model for the deck roughness. Attempts have been made to model the roughness in terms of statistical parameters from profile measurements of a large number of road or bridge surfaces. 


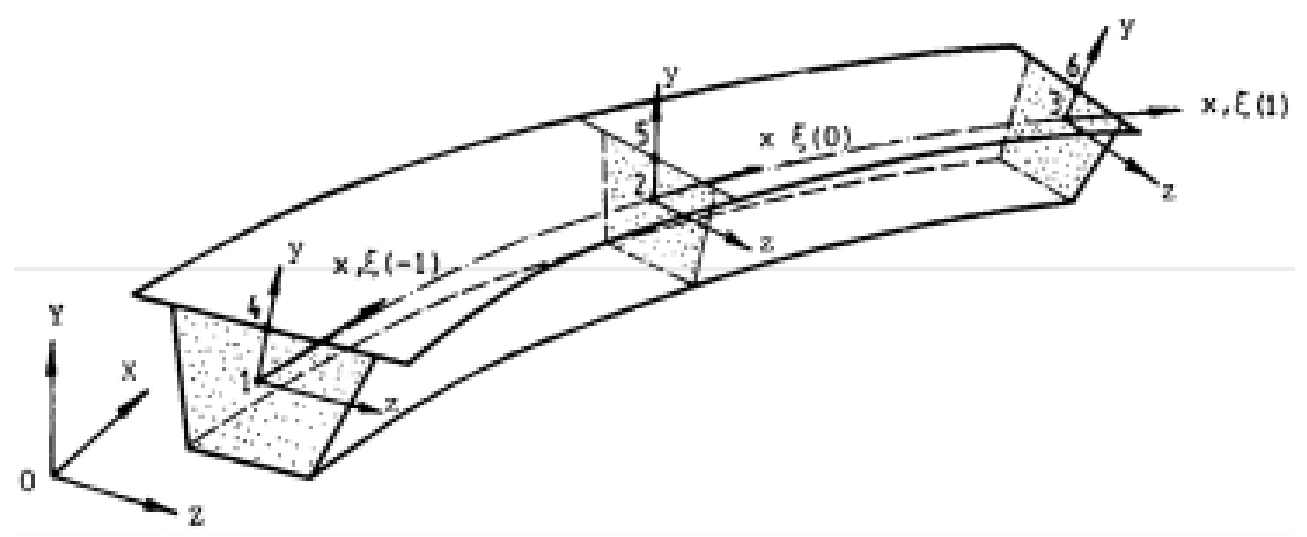

Fig. 2. Curved thin-walled box beam element with three nodes.

Honda et al. [13] showed that Power Spectral Density (PSD) could be related to parameters called roughness and waviness index. These parameters were used to shift the roughness level up and down and thus a guideline for classification of roads in terms of roughness were framed.

The vertical height of the bridge deck profile along the longitudinal axis measured with respect to flat datum at a distance $\mathrm{x}$ from the reference station can thus be represented as

$$
h(x)=h_{m}(x)+h_{r}(x)
$$

where $h_{m}(x)$ is the deterministic function describing the deck mean surface and $h_{r}(x)$ is a zero mean random process.

\subsubsection{Simulation of road roughness}

In the present study, random road input is specified in terms of PSD function. Vehicle response statistics for the given input can be obtained by using standard numerical integration scheme, with deck profile digitally simulated to be used as input.

The random road surface roughness $h_{r}(x)$ of the bridge can be described by zero mean, real-valued, stationary Gaussian process [14] as

$$
h_{r}(x)=\sum_{k=1}^{N} \alpha_{k} \cos \left(2 \pi \omega_{k}+\varphi_{k}\right)
$$

where $\alpha_{k}$ is the amplitude of the cosine wave, $\omega_{k}$, the frequency within the interval $\left[\omega_{l}, \omega_{u}\right]$ in which power spectral density function is defined, $\varphi_{k}$, the random phase angle with uniform probability distribution in the interval $[0,2 \pi]$. $x$ is the global coordinate measured left end of the bridge and $N$ is the total number of terms used to built up the road surface roughness. The value of $N$ depends on the velocity of the vehicle (hence the total time taken to cross the bridge) and size of the time increment chosen for the analysis of the dynamic response ( $N=$ Total time $/ \Delta t)$. The parameters $\alpha_{k}$ and $\omega_{k}$ are computed as

$$
\begin{aligned}
& \alpha_{k}^{2}=4 S_{r}\left(\omega_{k}\right) \Delta \omega \\
& \omega_{k}=\omega_{l}+(k-1 / 2) \Delta \omega \\
& \Delta \omega=\left(\omega_{u}-\omega_{l}\right) / N
\end{aligned}
$$

where $S_{r}\left(\omega_{k}\right)$ is the power spectral density function ( $\mathrm{m}^{3} /$ cycle), $\omega_{l}$ and $\omega_{u}$ are the lower and upper cut-off spatial frequencies (cycle/m), respectively.

The spectral density function $S_{r}\left(\omega_{k}\right)$ can be expressed in terms of the spatial frequency $\omega_{k}$, of the road surface roughness [15] as 
Table 1

Road surface classification

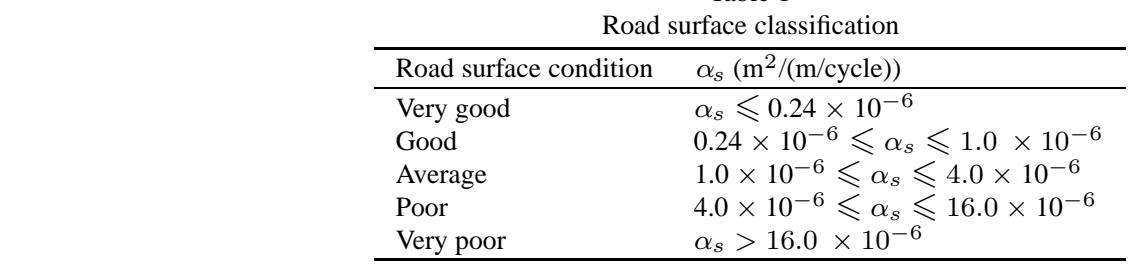

$$
S_{r}\left(\omega_{k}\right)= \begin{cases}\alpha_{s} \omega_{k}^{-\beta_{r}} & \text { for } \omega_{l}<\omega_{k}<\omega_{u} \\ 0 & \text { else where }\end{cases}
$$

where the parameter $\alpha_{s}$ is a spectral roughness coefficient in $\mathrm{m}^{2} /(\mathrm{m} / \mathrm{cycle})$ and the exponent $\beta_{r}$ is taken to be 1.94 . The road surface condition may be classified in to five classes according to ISO specification in terms of coefficient $\alpha_{s}$ as shown in the Table 1 .

\subsection{Bridge model}

A three-dimensional finite element analysis offers the most comprehensive treatment, where a variety of structural geometries, supports and loading conditions can be accommodated for the accurate assessment of structural effects. However, such an analysis is highly computing intensive and in some cases leads to voluminous data output. At the preliminary analysis and design stage, it is likely that a three-dimensional analysis may not be very reasonable since the bridge geometries and loading conditions, etc. may have to be modified for diverse reasons. It is, therefore, desirable at this stage to use a realistic, computationally efficient and less expensive model in the analysis.

The thin-walled box beam finite element developed by Zhang and Lyons [4], which is applicable for the analysis of single-spined multi-cell box girder bridges have been considered here for the dynamic analysis due to vehicle induced vibration. However, as per the records available, this element has not been used for the dynamic analysis. Thus, in the present work, an initial free vibration analysis has been carried out and the theoretical values of modal parameters have been verified experimentally. The subsequent application to complex bridge vehicle interaction studies have been carried out based on the satisfactory performance in the evaluation of modal parameters.

Although a continuous structure is in reality three-dimensional, a reduced one-dimensional form has certain simplifying advantages in many instances. However, the conventional truss or beam type elements are over simplified to represent thin-walled box girder bridges. The adopted thin-walled box beam element can be regarded as a general beam element. In addition to the usual six degrees of freedom at each node, represented by the three displacements and the three rotations, three more degrees of freedom have been incorporated in the formulation to account for the warping and distortion effects, which occur in box beams. The element is curved in space but the cross-sections are generated by straight lines. The element axis is defined as the locus of the centroids, which may be eccentric from but parallel to the flexural axis. The element has two end nodes and a midpoint node situated on the axis. The three noded thin-walled box beam element is as shown in Fig. 2. A local rectangular coordinates system $(x, y, z)$ along the curve axis is used in the element formulation. The global Cartesian coordinates are in terms of a natural coordinate $\xi$, which varies between -1 and +1 on the respective faces of the element.

The generalized displacements in the local co-ordinate system incorporating all the complexities of a thin-walled box girder are given by

$$
\bar{\delta}=\left[u, v, w, \theta_{x}, \theta_{y}, \theta_{z}, \theta_{x}^{\prime}, \gamma_{d}, \gamma_{d}^{\prime}\right]^{T}
$$

where $u, v, w$ are the translations along the local $x, y, z$ axes respectively, $\theta_{x}$ is the angle of twist, $\theta_{x}^{\prime}$ is the rate of twist, $\theta_{y}$ and $\theta_{z}$ are bending rotations about $y$ and $z$ axes respectively, $\gamma_{d}$ is the distortion angle, $\gamma_{d}^{\prime}$ is the rate of distortion.

The generalized stress vector is

$$
\sigma=\left[N_{x}, Q_{y}, Q_{z}, M_{T}, M_{y}, M_{z}, \frac{1}{\mu_{t}} B_{1}, M_{d}, B_{11}\right]^{T}
$$


in which $N_{x}$ is the axial force, $Q_{y}, Q_{Z}$ are the shear forces, $M_{T}$ is the pure torsional moment, $M_{y}, M_{z}$ are the primary bending moments, $B_{1}$ is the torsional warping bimoment, $M_{d}$ is the distortional moment, $B_{11}$ is the distortional warping bimoment and $\mu_{t}$ is the warping shear parameter.

The generalized strain vector is

$$
\begin{aligned}
& \varepsilon=\left[\varepsilon_{x}, \varepsilon_{y x}, \varepsilon_{z x}, \psi_{\theta x}, \psi_{y x}, \psi_{z x}, \psi_{w t x}, \psi_{d x}, \psi_{w d x}\right]^{T} \\
& \text { where, axial strain } \varepsilon_{x}=\frac{\partial u}{\partial x}
\end{aligned}
$$

Shear strain in $y-\operatorname{direction} \varepsilon_{y x}=\frac{\partial v}{\partial x}+\frac{\partial u}{\partial y}=\frac{\partial v}{\partial x}-\theta_{z}$

Shear strain in $z-\operatorname{direction} \varepsilon_{z x}=\frac{\partial w}{\partial x}+\frac{\partial u}{\partial z}=\frac{\partial w}{\partial x}+\theta_{y}$

Torsional strain $\psi_{\theta x}=\frac{\partial \theta_{x}}{\partial x}$

Flexural strain about $y-$ axis $\psi_{y x}=\frac{\partial^{2} u}{\partial x \partial z}=\frac{\partial \theta_{y}}{\partial x}$

Flexural strain about $z-$ axis $\psi_{z x}=\frac{\partial^{2} u}{\partial x \partial y}=-\frac{\partial \theta_{z}}{\partial x}$

Torsional warping strain $\psi_{\omega t x}=-\frac{\partial^{2} \theta_{x}}{\partial x^{2}}-\frac{1}{R} \frac{\partial \theta_{z}}{\partial x}$

$R$ is the radius of curvature and the modification in the torsional warping strain has been introduced to take into account the effect caused by the initial curvature.

Distortional strain $\psi_{d x}=\gamma_{d}$

Distortional warping strain $\psi_{\omega d x}=\frac{-\partial^{2} \gamma_{d}}{\partial x^{2}}$

A modification has been made to the torsional warping strain to take into account the effect caused by the initial curvature.

The generalized elasticity matrix is

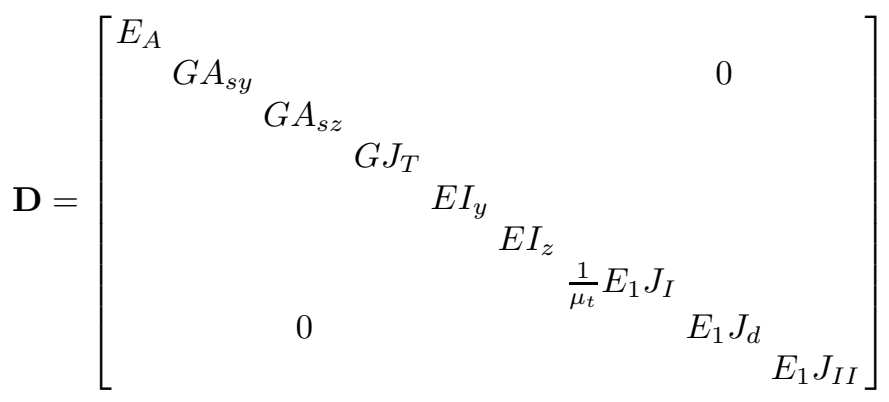

where $A$ is cross-sectional area; $A_{s y}, A_{s z}$, are effective shear areas in the $y$ and the $z$ directions respectively; $J_{T}$ is torsional moment of inertia; $I_{y}, I_{z}$ are bending moments of inertia about the $y$ and $z$ axes respectively; $J_{I}$ is torsional warping moment of inertia; $J_{d}$ is distortional second moment of area; and $J_{I I}$ is distortional warping moment of inertia. $E_{1}$ is the conversion modulus of elasticity and is given by

$$
E_{1}=\frac{E}{\left(1-\nu^{2}\right)}
$$


where $\mathrm{E}$ and $\mu$ are the Young's modulus of elasticity and Poisson's ratio. Further, the bending moments of inertia have been calculated on the basis of an effective flange breath replacing the actual width to account for the effect of shear lag. Only $C_{0}$ continuity is required for the extensional and flexural effects and quadratic shape functions have been used

$$
\begin{array}{ll}
N_{i}=\frac{1}{2}\left(\xi^{2}+\xi_{0}\right) & \text { for } i=1 \text { and } 3 \\
N_{i}=\left(1-\xi^{2}\right) & \text { for } i=2
\end{array}
$$

However, since the governing equations for torsion and distortion are of fourth order and the beam being threenoded, fifth order $C_{1}$ continuity is required for torsion and distortion.

$$
\begin{aligned}
& N_{i 1}=\left(\frac{\xi^{2}}{4}\right)\left(4+5 \xi_{0}-2 \xi^{2}-3 \xi_{0}^{3}\right) \text { for } i=1 \text { and } 3 \\
& N_{i 2}=\left(\frac{J_{i}}{4}\right) \xi^{2}\left(1-\xi_{0}\right)\left(1-\xi^{2}\right) \\
& N_{i 1}=\left(1-\xi^{2}\right)^{2} \text { for } i=2 \\
& N_{i 2}=J_{i} \xi\left(1-\xi^{2}\right)^{2}
\end{aligned}
$$

where $\xi_{0}=\xi \xi_{i}$ and $J_{i}$ is the Jacobian factor with respect to nodal coordinates.

The element stiffness matrix may be written as

$$
\mathrm{k}^{e}=\int_{-l / 2}^{l / 2} \mathrm{~B}^{T} \mathrm{DB} d x=\int_{-1}^{+1} J \mathrm{~B}^{T} \mathrm{DB} d \xi
$$

The nodal force vector equivalent to internal and external forces is written as

$$
\mathbf{f}^{e}=\int_{l} \mathbf{N}^{T} b d x+\int_{l} \mathbf{N}^{T} q d x+\sum \mathbf{N}^{T} P+\int_{l} \mathbf{B}^{T} \mathrm{D} \varepsilon_{0}-\int_{l} \mathbf{B}^{T} \sigma_{0} d x
$$

where $\mathbf{B}$ is the strain displacement matrix, $b$ is body force vector, $q$ is distributed force vector, $P$ is concentrated force vector, $\varepsilon_{0}$ is initial strain vector including temperature effects, and $\sigma_{0}$ is initial stress vector.

Exact integration of Eq. (24) is achieved by using a three-point Gauss quadrature for the axial and bending contributions and a six-point Gauss quadrature for the torsional and distortional contributions. However, to eliminate the spurious shear effects associated with beam formulations which include shear deformations, a two-point Gauss quadrature is used for axial and bending contributions.

\section{Equations of motion for coupled Bridge-Vehicle system}

The equation of motion of the sprung mass can be written as

$$
m_{s} \ddot{z}+\sum_{i=1}^{2} \sum_{p=1}^{2}\left\{c s_{i p}\left(\dot{z}-b_{i} \dot{\theta}-l_{p} \dot{\psi}-\dot{z}_{i p}\right)+k s_{i p}\left(z-b_{i} \theta-l_{p} \psi-z_{i p}\right)\right\}=0
$$

As the random input of deck profile is not same for the front and rear wheels, the vehicle is subjected to pitching. The pitching motion of sprung mass is given by

$$
J \ddot{\psi}+\sum_{i=1}^{2} \sum_{p=1}^{2}\left\{-c s_{i p}\left(\dot{z}-b_{i} \dot{\theta}-l_{p} \dot{\psi}-\dot{z}_{i p}\right) l_{p}-k s_{i p}\left(z-b_{i} \theta-l_{p} \psi-z_{i p}\right) l_{p}\right\}=0
$$




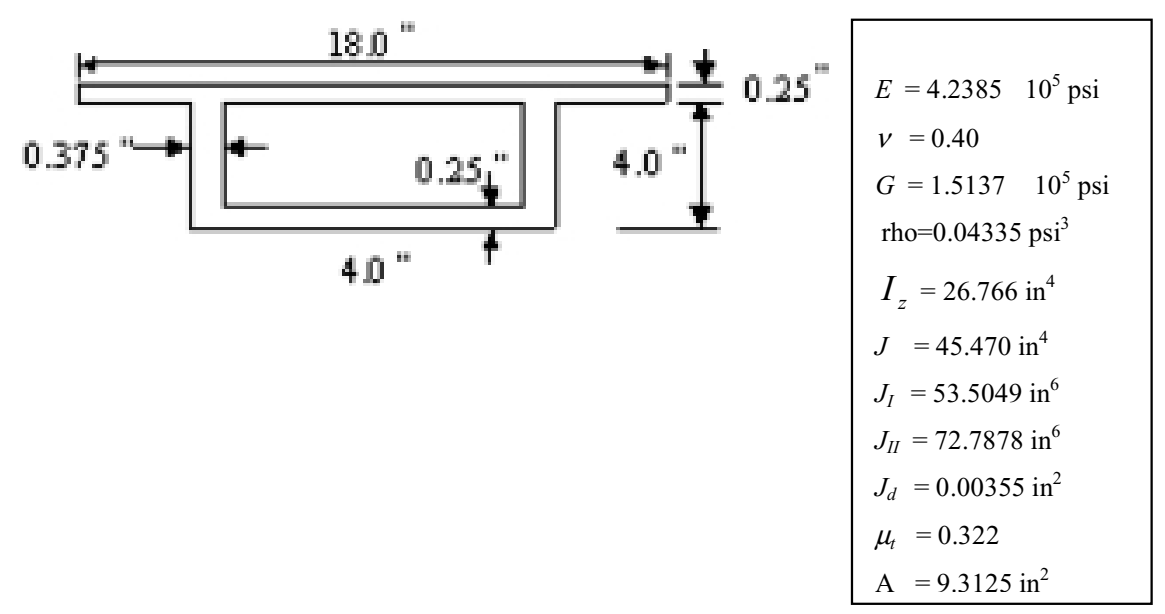

Fig. 3. Geometry (inch) and properties of the box girder model.

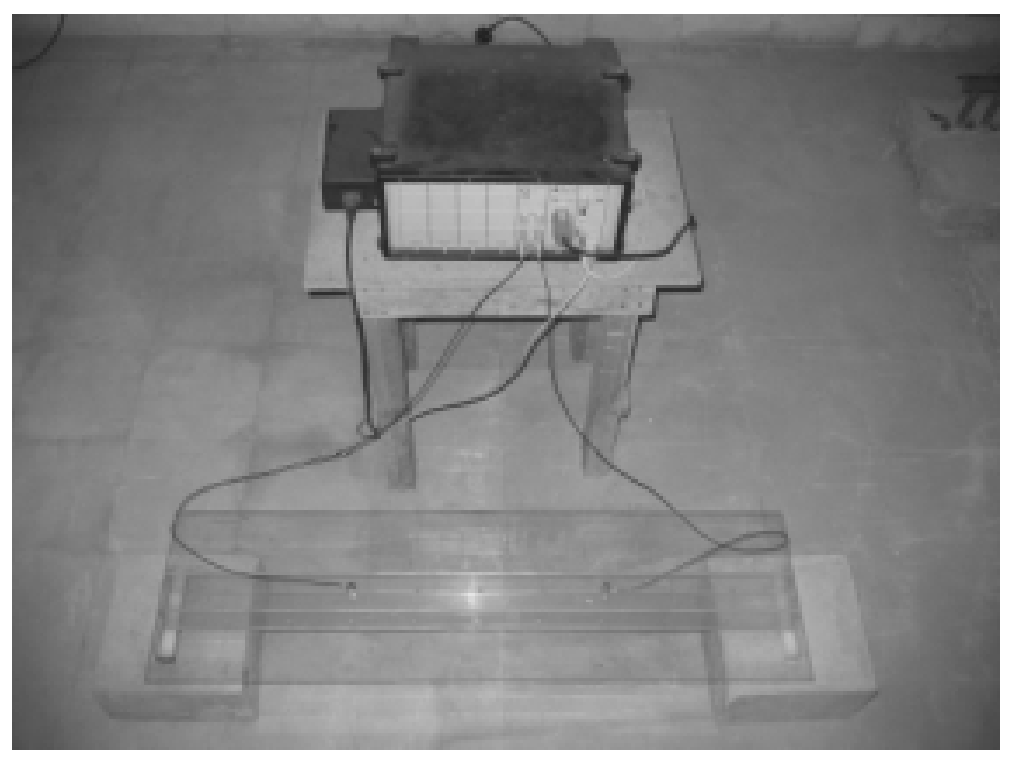

Fig. 4. Experimental setup for free vibration study of a simply supported curved box girder bridge model.

The rolling motion of sprung mass is given by

$$
I \ddot{\theta}+\sum_{i=1}^{2} \sum_{p=1}^{2}\left\{-c s_{i p}\left(\dot{z}-b_{i} \dot{\theta}-l_{p} \dot{\psi}-\dot{z}_{i p}\right) b_{i}-k s_{i p}\left(z-b_{i} \theta-l_{p} \psi-z_{i p}\right) b_{i}\right\}=0
$$

The front and rear wheel bounce can be represented as

$$
\begin{aligned}
& m_{i p} \ddot{z}_{i p}-c s_{i p}\left(\dot{z}-b_{i} \dot{\theta}-l_{p}-\dot{z}_{i p}\right)-k s_{i p}\left(z-b_{i} \theta-l_{p}-z_{i p}\right) \\
& +c u_{i p}\left(\dot{z}_{i p}-\dot{h}_{i p}-\dot{v}_{i p}\right)+k u_{i p}\left(z_{i p}-h_{i p}-v_{i p}\right)=0 \\
& i=1,2 ; p=1,2 ;
\end{aligned}
$$

The governing differential equation of motion of the box-girder bridge can be expressed as 


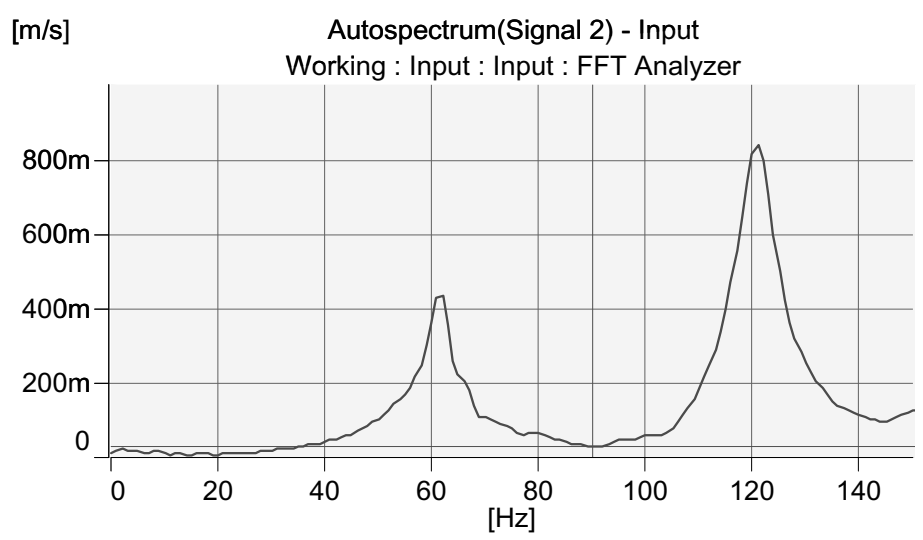

Fig. 5. Frequency response function plot for the simply supported curved box girder bridge model.

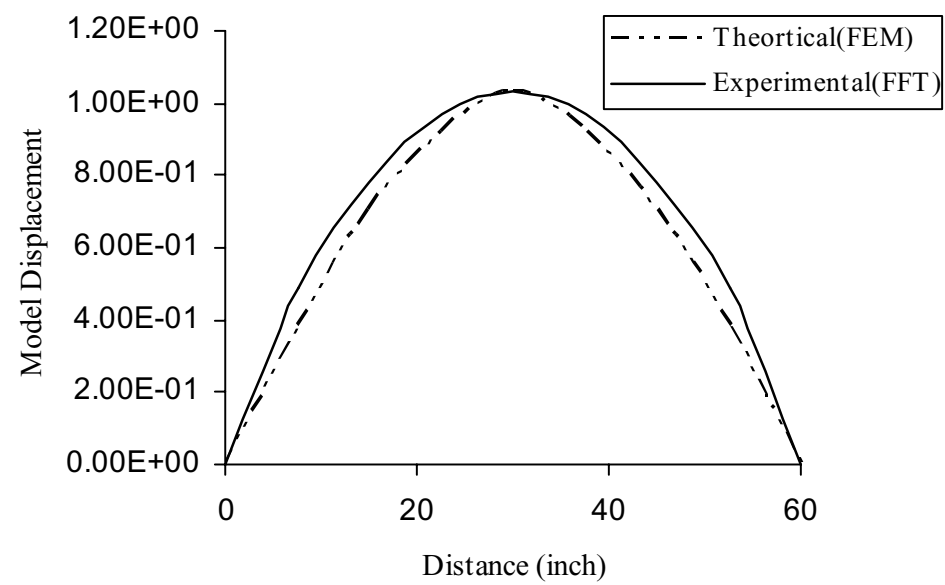

Fig. 6. 1st mode (flexural) shape of curved box girder.

$$
\begin{aligned}
& {\left[m_{b}\right]\{\ddot{\delta}\}+\left[c_{b}\right]\{\dot{\delta}\}+\left[k_{b}\right]\{\delta\}-\sum_{i=1}^{2} \sum_{i=1}^{2}\left\{c u_{i p}\left(\dot{z}_{i p}-\dot{h}_{i p}-\dot{v}_{i p}\right)+k u_{i p}\left(z_{i p}-h_{i p}-v_{i p}\right)\right\}} \\
& +\left(m_{s} g+\sum_{i=1}^{2} \sum_{p=1}^{2} m_{i p} g\right)+\left(\frac{m_{s} v^{2}}{R}+\sum_{i=1}^{2} \sum_{p=1}^{2} \frac{m_{i p} v^{2}}{R_{i}^{\prime}}\right)=0
\end{aligned}
$$

The weight of the vehicle and centrifugal forces will also act at appropriate location in addition to the damping and spring forces from vehicle as shown in Eq. (30).

Here, $b_{1}=a_{3} s_{2}, b_{2}=a_{4} s_{2}, l_{1}=a_{1} s_{1}, l_{2}=a_{2} s_{1}$ and $b_{2}, l_{2}$ are negative quantities. $v_{i p}$ are the bridge displacements under front/rear wheels at any arbitrary time $t . h_{i p}$ represents the random input of deck profile under the front/rear wheels and $\dot{h}_{i p}$ are the time derivatives of the random input of deck profile.

The suspension stiffness and damping of the vehicle are denoted by $k s_{i p}$ and $c s_{i p}$. Similarly, the tyre stiffness and damping are denoted by $k u_{i p}$ and $c u_{i p}$. The unsprung mass is denoted by $m_{i p}$, which corresponds to tyre mass and $m_{s}$ represents the sprung mass. The Moment of inertia for pitch and roll of the vehicle are designated by $J$ and $I$, while $\left[m_{b}\right],\left[c_{b}\right],\left[k_{b}\right]$ represents the bridge mass, damping and stiffness matrices respectively and $g$ is the acceleration due to gravity. $R$ is the radius of curvature with respect to the center of gravity of vehicle and $R_{i}^{\prime}$ is the corresponding radius of curvature with respect to the right and left wheel. The tyres of the vehicle always remain in contact with the bridge deck and the coupled bridge-vehicle system is solved for the range of velocities such that the interaction force is never less than zero. Newmark- $\beta$ scheme (average acceleration) with predictor-corrector algorithm [16] has been used for the evaluation of dynamic response of the bridge due to vehicle-induced vibration. 


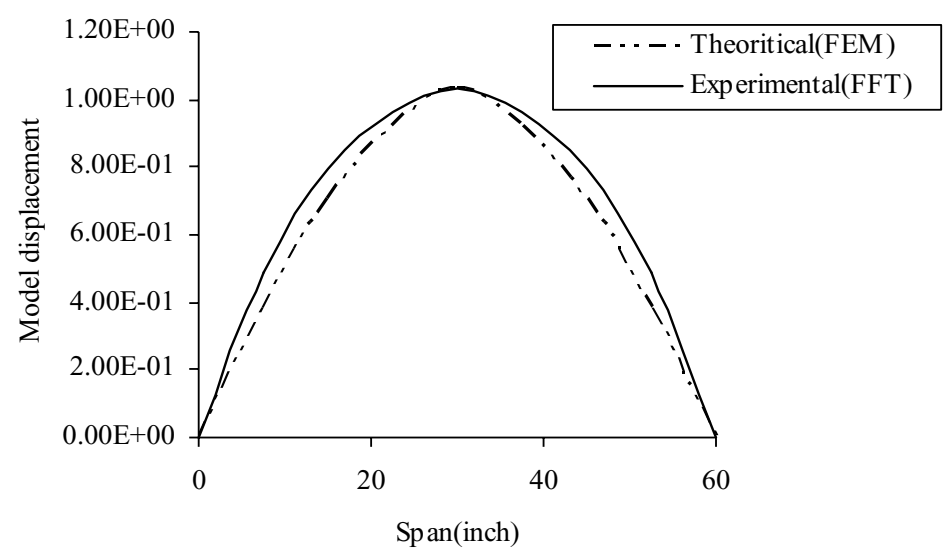

Fig. 7. 2nd mode (lateral) shape of curved box girder.

\section{Numerical simulation}

Free vibration studies have been conducted prior to the evaluation of dynamic response of curved thin-walled box girder bridge due to vehicle-induced vibration. The natural frequencies and mode shapes are obtained experimentally and compared with the theoretical values, which are obtained using the adopted finite element model proposed by Zhang and Lyons [4].

\subsection{Free vibration}

A simply supported curved box girder model fabricated from perspex sheets as mentioned in Ref. [17] has been considered for the free vibration analysis in the present study. The perspex sheets model has been used for the experimental as well theoretical evaluation of modal parameters. The span of the model considered is 60 in $(1.52 \mathrm{~m})$ with the radius of curvature of 1200 in $(30.48 \mathrm{~m})$. The cross-sectional dimension is as shown in Fig. 3.

First two frequencies for the simply supported curved box girder model have been determined experimentally by picking up responses through the accelerometers (sensitivity $10 \pm 2 \% \mathrm{mV} / \mathrm{ms}^{-2}$ ) mounted on the curved box girder model along the center line and processed by the Bruel and Kjaer FFT analyzer with an in-built PULSE software. These two modes correspond to vertical and lateral bending of the bridge specimen. The experimental setup for the evaluation of modal parameters of the simply supported curved box girder bridge model is as shown in Fig. 4. The natural frequencies for the simply supported curved box girder model during an impact excitation could be observed accurately for the first two modes and hence results are presented for the first two modes. Figure 5 shows a Frequency Response Function (FRF) obtained experimentally. The peaks of the FRF correspond to the natural frequencies of the bridge being tested. The variation between theoretical and experimental results of the first two frequencies have been presented in Table 3. The modes shapes as obtained theoretically as well as from experimental observation [18], have been presented in Figs 6 and 7. The agreement obtained has been observed to be quite satisfactory. Thus, the three noded thin-walled box beam finite element model can be applied reliably for the evaluation of forced vibration response of the box girder bridge due to vehicle-induced excitation.

\subsection{Vehicle induced vibration}

It is assumed that the damping matrix of the bridge is proportional to mass and stiffness, which is taken into account by assuming Rayleigh damping. One percent of critical damping is taken for the first and second modes. It is assumed that the bridge surfaces have the same roughness in the transverse direction. It is also assumed that the roadway approaches also have the same class of road surface as the bridge. 
Table 2

Data of vehicle with seven degrees of freedom (Fig. 1)

\begin{tabular}{lcc}
\hline Parameter & Unit & Value \\
\hline Sprung mass $\left(m_{s}\right)$ & $\mathrm{Kg}$ & 15000 \\
Unsprung mass in front axle $\left(m_{11}, m_{21}\right)$ & $\mathrm{kg}$ & 800 \\
Unsprung mass in rear axle $\left(m_{12}, m_{22}\right)$ & $\mathrm{kg}$ & 710 \\
Vehicle suspension stiffness $\left(k s_{i p}\right)$ & $\mathrm{N} / \mathrm{m}$ & $0.399 \times 10^{6}$ \\
Vehicle tyre stiffness $\left(k u_{i p}\right)$ & $\mathrm{N} / \mathrm{m}$ & $0.351 \times 10^{6}$ \\
vehicle suspension damping in front axle $\left(c s_{11}, c s_{21}\right)$ & $\mathrm{Ns} / \mathrm{m}$ & 23210 \\
vehicle suspension damping in rear axle $\left(c s_{12}, c s_{22}\right)$ & $\mathrm{Ns} / \mathrm{m}$ & 5180 \\
Vehicle tyre damping $\left(c u_{i p}\right)$ & $\mathrm{Ns} / \mathrm{m}$ & 800 \\
Pitch moment of inertia $(J)$ & $\mathrm{kgm}{ }^{2}$ & 154.536 \\
Roll moment of inertia $(I)$ & $\mathrm{kgm}{ }^{2}$ & 449 \\
Position parameter (length wise) $a_{1}$ & $\mathrm{~m}$ & 0.35 \\
Position parameter (length wise) $a_{2}$ & $\mathrm{~m}$ & 0.65 \\
Position parameter (breath wise) $a_{3}$ & $\mathrm{~m}$ & 0.5 \\
Position parameter (breath wise) $a_{4}$ & $\mathrm{~m}$ & 0.5 \\
Vehicle axle spacing (length wise) $s_{1}$ & $\mathrm{~m}$ & 2.66 \\
Vehicle axle spacing (breath wise) $s 2$ & $\mathrm{~m}$ & 1.5 \\
Height of C.G. of vehicle from deck surface $\left(h_{v}\right)$ & $\mathrm{m}$ & 1.2 \\
\hline
\end{tabular}

Table 3

Structural frequency of box girder bridge

\begin{tabular}{lccc}
\hline Frequency (Hz) & Theoretical (FEM) & Experimental (FFT) & \% Error \\
\hline First mode(flexural) & 59.1726 & 61.50 & 3.93 \\
Second mode(lateral) & 116.2049 & 121.00 & 3.96 \\
\hline
\end{tabular}

\subsubsection{Vehicle parameters}

The vehicle model as mentioned in Section 2.1 is a heave-pitch-roll 3D model. All the parameters relevant to the vehicle are taken from ref. [19] and have been presented in Table 2.

\subsubsection{Road roughness profile model}

The details of the procedure for generation of random road surface roughness from PSD function have been given in the Section 2.2.1. In this study, the values of spectral roughness coefficient, $\alpha_{s}$ have been taken as $0.24 \times 10^{-6}$, $0.5 \times 10^{-6}, 3.0 \times 10^{-6}, 10.0 \times 10^{-6}$ and $25.0 \times 10^{-6} \mathrm{~m}^{3} /(\mathrm{m} / \mathrm{cycle})$ according to International Organization for Standardization (ISO) specifications for the classes of very good, good, average, poor and very poor roads respectively. Twenty profiles of road roughness are generated for each type of road using the following parameters:

The lower and upper limits of the spatial frequencies of the road profile are taken as $\omega_{l}=0.01 \mathrm{cycle} / \mathrm{m}$ and $\omega_{u}=3.0$ cycle $/ \mathrm{m}$. The cut-off spatial frequencies are chosen in view of the practical size of a tyre. In generating the random road surface roughness, random numbers $\phi_{k}$ have been generated in MATLAB. Thus, for the bridge deck span of $30 \mathrm{~m}$, deck profile heights are generated along the span of the bridge at discrete points, which corresponds to the velocity of the vehicle and time step size chosen. A typical vertical highway surface profile of good road surface is as shown in Fig. 9. Dynamic responses are evaluated for each of the simulations corresponding to a particular vehicle velocity.

\subsubsection{Bridge model}

A simply supported curved box girder bridge (Fig. 8) as considered by Kermani [2] has been chosen in the present study to obtain the dynamic characteristics. The span of the simply supported bridge is $30 \mathrm{~m}$ with a radius of curvature of $150 \mathrm{~m}$ and the bridge has diaphragms at supports. The mass density, modulus of elasticity and Poisson's ratio of the material are $2403 \mathrm{~kg} / \mathrm{m}^{3}, 3.45 \times 10^{10} \mathrm{~N} / \mathrm{m}^{2}$ and 0.15 respectively. Though the chosen Newmark- $\beta$ scheme is unconditionally stable, all the analyses have been carried out by choosing a time increment equal to $1 / 50^{\text {th }}$ of first fundamental time period from the accuracy point of view. Further, more refined time increments values have also been considered, which did not show any significant changes in the dynamic response of the bridge under study. Thus, considering numerical error due to inappropriate space and time discretizations, the bridge has been discretized using thirty numbers of thin-walled box beam elements and a time step of $3.6 \times 10^{-3} \mathrm{sec}$ is chosen for the analysis. 
Table 4

Effect of damping on impact factor

\begin{tabular}{cccccc}
\hline \multicolumn{7}{c}{ Damping ratio $(\xi)$} \\
\hline Response parameter & $0 \%$ & $1 \%$ & $2 \%$ & $3 \%$ & $4 \%$ \\
\hline$\left(M_{z}\right)$ & 1.13 & 1.08 & 1.07 & 1.05 & 1.03 \\
$\left(Q_{Y}\right)$ & 1.15 & 1.08 & 1.07 & 1.06 & 1.05 \\
$\left(M_{x}\right)$ & 2.05 & 1.93 & 1.92 & 1.92 & 1.92 \\
$\left(B_{I}\right)$ & 2.25 & 2.24 & 2.24 & 2.24 & 2.24 \\
$\left(M_{D}\right)$ & 1.53 & 1.52 & 1.52 & 1.51 & 1.51 \\
$\left(B_{I I}\right)$ & 1.80 & 1.79 & 1.79 & 1.79 & 1.78 \\
$(v)$ & 1.21 & 1.14 & 1.13 & 1.11 & 1.09 \\
\hline
\end{tabular}

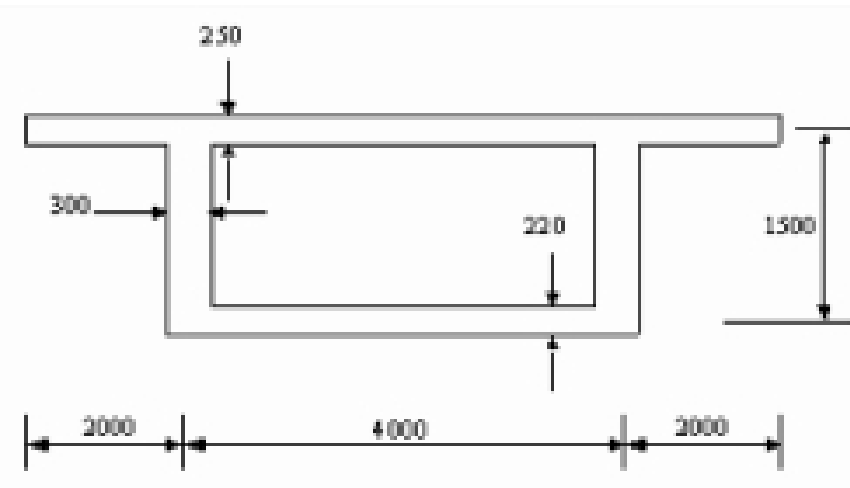

\begin{tabular}{|l|}
$E=3.45 \mathrm{e}+10 \mathrm{~N} / \mathrm{m}^{2}$ \\
$v=0.15$ \\
$G=1.5 \mathrm{e}+10 \mathrm{~N} / \mathrm{m}^{2}$ \\
$I_{z}=1.6061 \mathrm{~m}^{4}$ \\
$J=3.2593 \mathrm{~m}^{4}$ \\
$J_{I}=0.79304 \mathrm{~m}^{6}$ \\
$J_{I I}=1.01304 \mathrm{~m}^{6}$ \\
$J_{d}=0.00563 \mathrm{~m}^{2}$ \\
$\mu_{t}=0.365$ \\
$\mathrm{~A}=3.7050 \mathrm{~m}^{2}$
\end{tabular}

Fig. 8. Geometry (mm) and properties of box girder bridge.

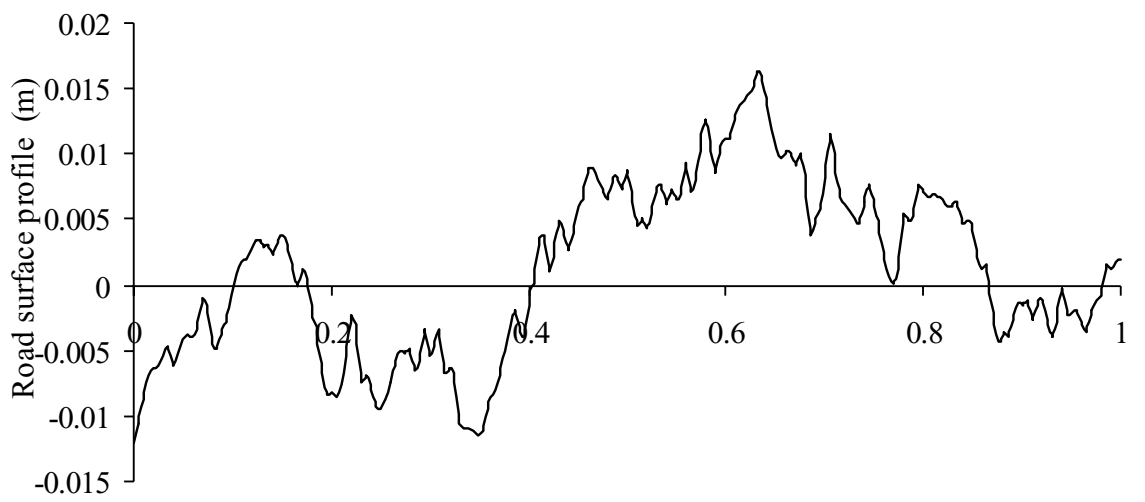

$\mathrm{x} / \mathrm{L}$

Fig. 9. A typical good road surface profile for a box girder bridge.

\subsubsection{Typical of time histories of the box girder bridge response}

Some typical time histories for the bridge (Fig. 8) are shown in Figs 10-16. The histories are obtained at the mid span of the curved thin-walled box-girder bridge corresponding to a good road surface roughness with a vehicle speed of $20 \mathrm{~m} / \mathrm{sec}$ and asymmetric car loading (Fig. 17, loading case 2) as the vehicle crosses the bridge. The abscissa in those time histories is the distance measured from the left of the bridge to the front axle of the vehicle. Figures 10 and 11 show the time histories at the mid span of the bridge for the bending moment about the transverse axis and the deflection along the vertical direction at the center of the bridge as the vehicle moves along the span. 


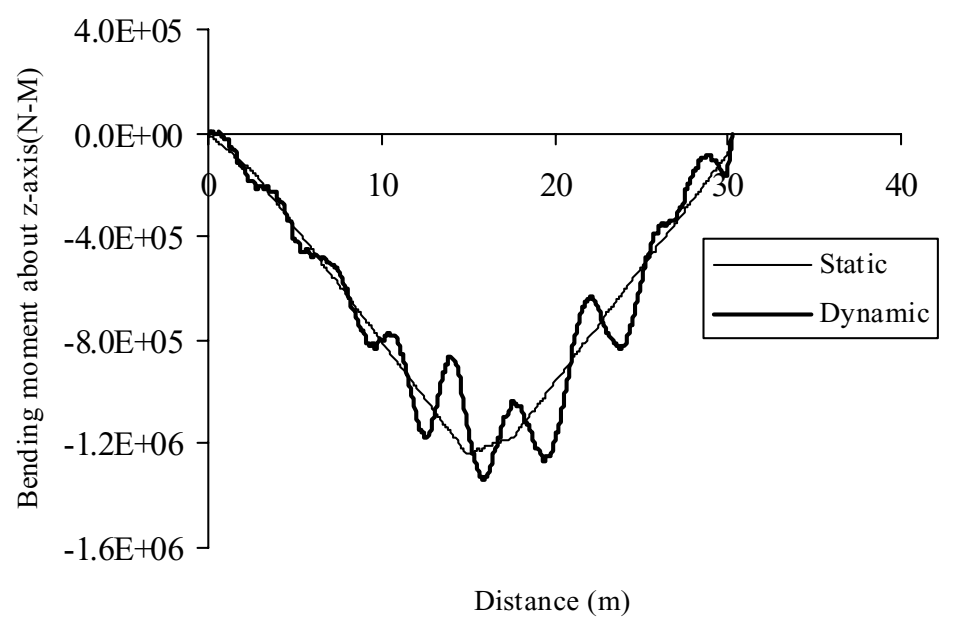

Fig. 10. Time histories for bending moment about transverse axis.

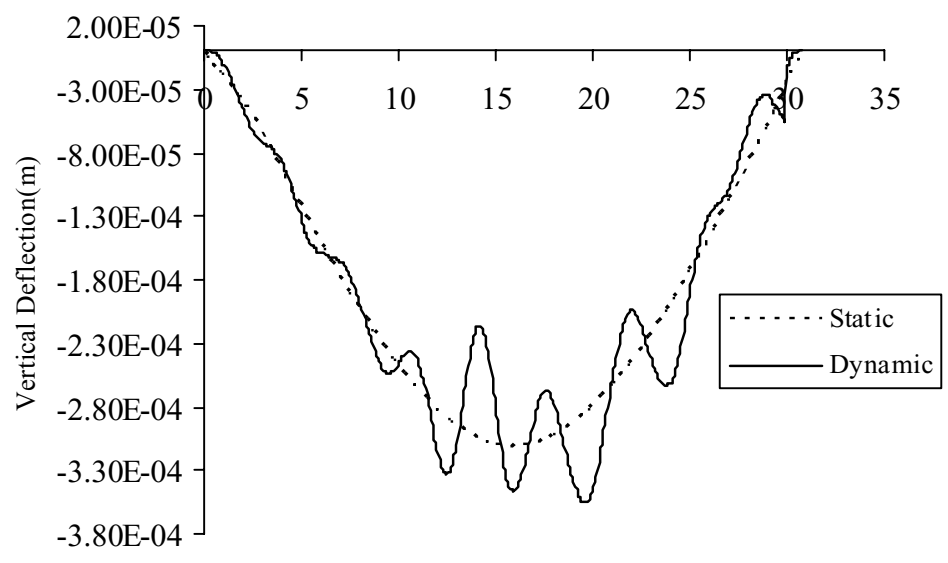

Distance (m)

Fig. 11. Time histories for vertical deflection.

From this figures, it can be observed that the vibration modes with higher frequencies significantly contributes to the dynamic response. Figures 12 and 13 show the histories of shear force and torsional moment at the center of the bridge. These figures indicate that the centrifugal force has greatly influenced the dynamic response of torque, while the shear force is almost unaffected by centrifugal force. Figures 14-16 show the time histories of torsional bimoment, distortional moment and distortional bimoment. A significant effect of higher frequency modes as well as centrifugal forces on those dynamic responses have been observed. Thus, similar to the established findings in the existing literature, it has been clearly observed from Figs 10-16 that a substantial difference exist in all the response parameters between static and dynamic loading conditions. The impact factor have been suitably evaluated corresponding to each of these parameters.

\subsection{Impact factor}

In the present study, Impact factor is defined as the ratio of absolute maximum dynamic and static responses corresponding to the $j^{t h}$ simulation of random road surface. The impact factor usually adopted in design practices does not depend on time. Hence the largest absolute value of the impact factor calculated for a particular section has been presented in the report. Effects of various parameters such as damping of box-girder structure, load positions, 


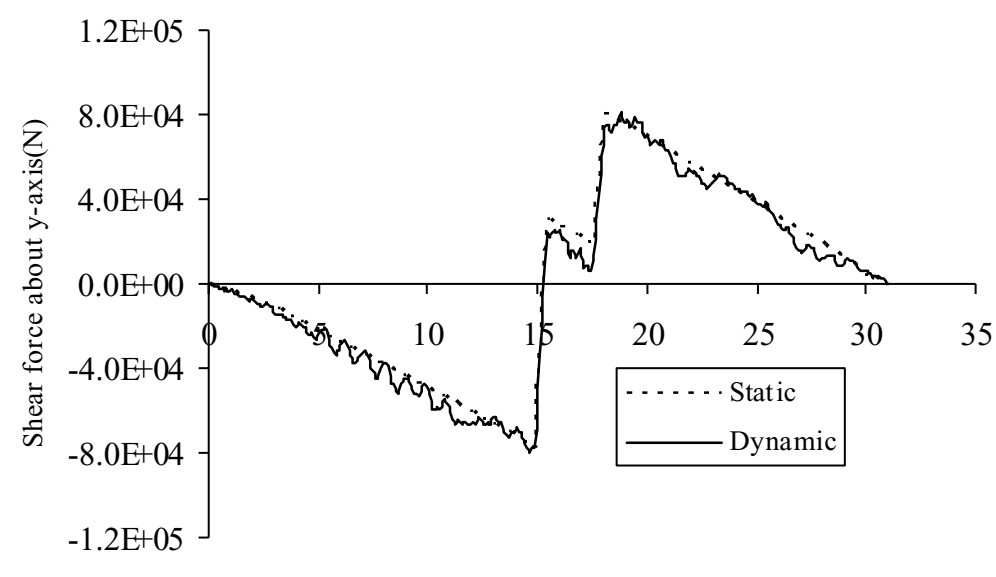

Distance (m)

Fig. 12. Time histories for shear force.

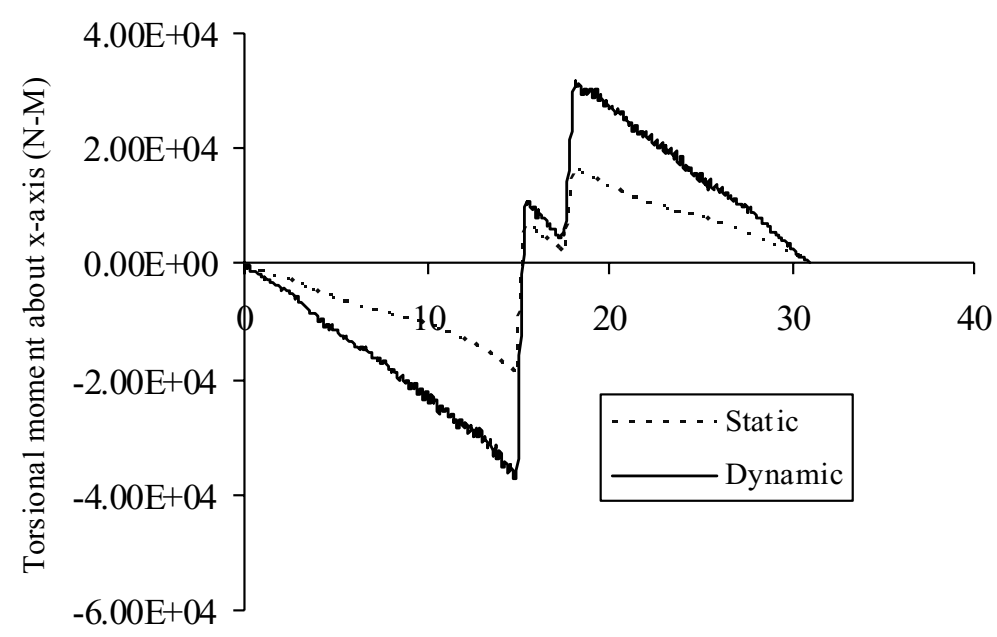

Distance (m)

Fig. 13. Time histories for torsional moment.

vehicle speed, span, radius of curvature of the box girder bridge, surface roughness, suspension stiffness and sprung mass on the impact factor discussed in this section. The impact factor as presented in Tables 4-12 represents the mean values corresponding to twenty simulation of road surface corresponding to a particular type of road condition.

The example problem as shown in Fig. 7 with a span length of $30 \mathrm{~m}$ and a radius of curvature of $150 \mathrm{~m}$ with a good road surface roughness has been considered again. A vehicle speed of $20 \mathrm{~m} / \mathrm{sec}$ and an asymmetric car loading (Fig. 17, loading case 2) has been chosen. The damping ratio for the bridge is taken as $1 \%$. However, some of these physical parameters will be varied in order to evaluate the influence of them on impact factors based on different response parameters.

\subsubsection{Parameter study}

The influence of certain physical parameters on the Bending moment about the transverse axis $\left(M_{z}\right)$, Shear force $\left(Q_{Y}\right)$, Torsional moment $\left(M_{x}\right)$, Torsional Bimoment $\left(B_{I}\right)$, Distortional moment $\left(M_{D}\right)$, Distortional Bimoment $\left(B_{I I}\right)$, Vertical deflection $(v)$ have been studied in details here. It is fully appreciated that all these response parameters are highly significant for a thin-walled box girder bridge. Thus, the impact factor based on these response 


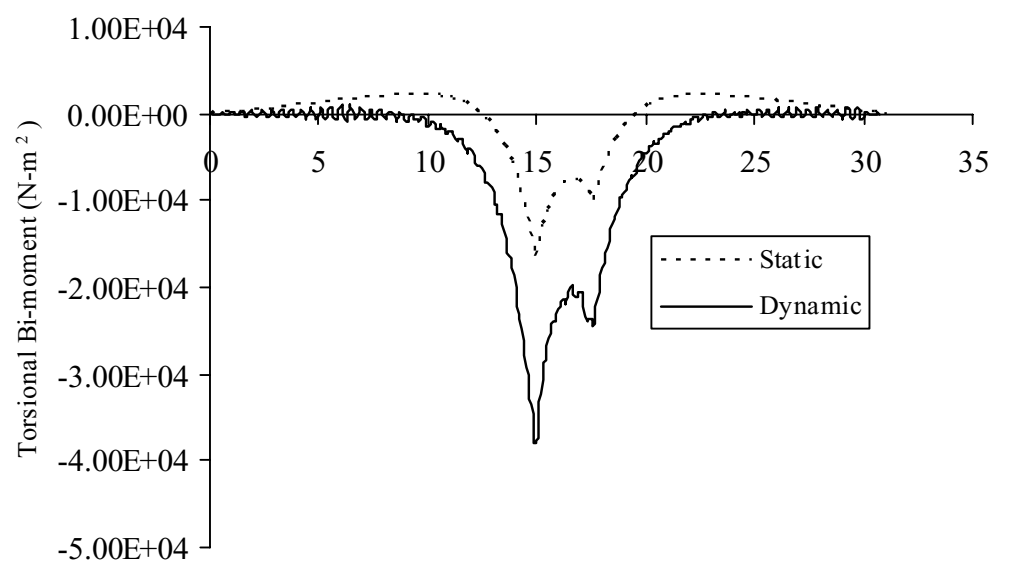

Distance $(\mathrm{m})$

Fig. 14. Time histories for torsional bimoment.

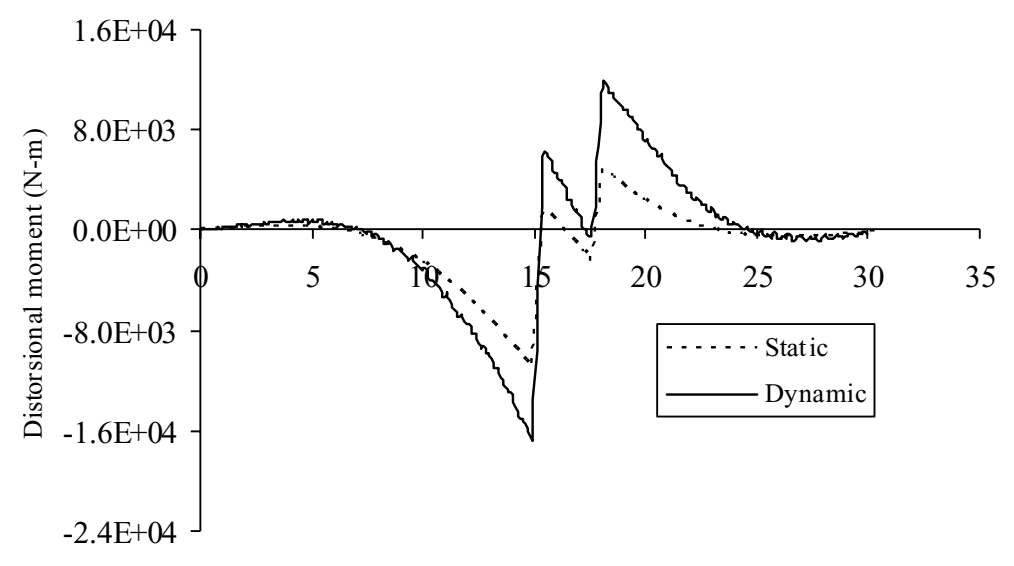

Distance (m)

Fig. 15. Time histories for distortional moment.

parameters will also accordingly reflect the significance of all the physical parameters considered for the present study.

(i) Effect of damping

Table 4 shows the variation of impact factor corresponding to various response parameters and varying damping ratio considered in the study. It has been observed that neglecting the effect of damping will reasonably overestimates the impact factors of vertical deflection, bending moment and shear force for a curved box-girder bridge. However, with increasing damping ratio from $1 \%$ to $4 \%$, only small decrease of impact factors have been observed.

(ii) Effect of load positions

In order to find the most unfavourable loading position for the assessment of impact factor, six different positions of traverse load on a box-girder bridge have been considered as shown in Fig. 17 in line with ref. [7]. Table 5 shows the variation of impact factor corresponding to different response parameters with different loading cases. The impact factors of curved box-girder bridge for a two- truck loading have been observed to be higher than that for a single truck loading. The loading positions have lesser influence on vertical shear and bending moment, while impact factors of torque, distortional moments and bimoments are relatively more effected by the loading positions.

(iii) Effect of vehicle speed

Table 6 gives the variation of impact factor with vehicle speed. The table shows that while the impact factors 


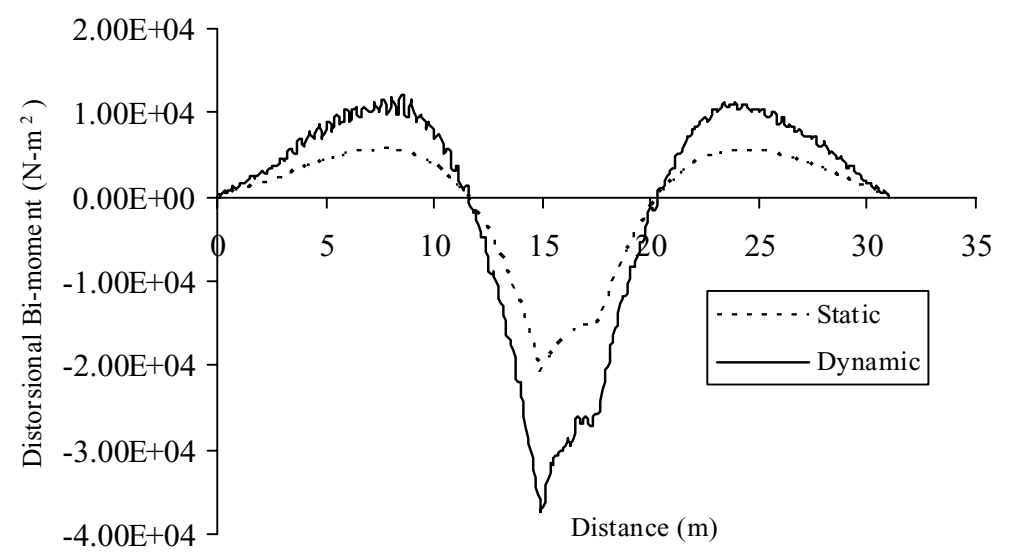

Fig. 16. Time histories for distortional bimoment.

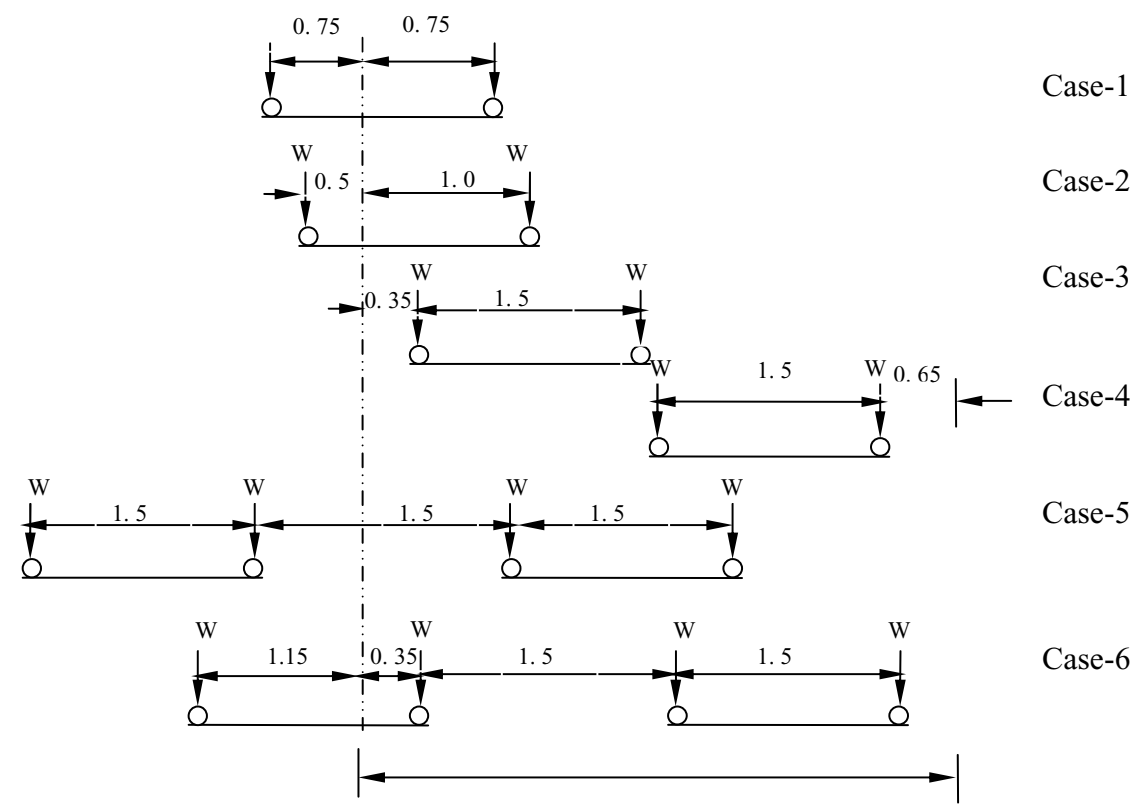

Half width of bridge

Fig. 17. Traverse loading cases on curved box girder bridge.

of torque, distortion and their corresponding bi-moments rapidly increase with vehicle speed, those of vertical deflection, bending moment and shear force do not show such tendency.

(iv) Effect of span of box girder bridge

Table 7 shows the influence of span of curved box girder bridge on the impact factors based on different response parameters. It can be observed that the impact factors of all the responses decrease with the increase of span length. However, the variation of the impact factor of torsion, distortion and their corresponding bimoments are comparatively significant, while those of the vertical deflection, bending moment and shear force are almost unaffected for the range of span data considered in the present study.

(v) Effect of radius of curvature of box girder bridge

Table 8 shows the variation of impact factor with the varying radius of curvature of box-girder bridge. The table shows that the impact factors corresponding to different response parameters of box-girder bridges decrease with the 
Table 5

Effect of loading position on impact factor

\begin{tabular}{cccccccc}
\hline \multicolumn{9}{c}{ Loading case } \\
\hline Type of truck & \multicolumn{9}{c}{ Single truck } & & \multicolumn{2}{c}{ Double truck } \\
\cline { 2 - 5 } Response parameter & Case-1 & Case-2 & Case-3 & Case-4 & & Case-5 & Case-6 \\
\hline$\left(M_{z}\right)$ & 1.08 & 1.08 & 1.0960 & 1.10 & & 1.56 & 1.56 \\
$\left(Q_{Y}\right)$ & 1.08 & 1.08 & 1.0552 & 1.05 & & 1.45 & 1.45 \\
$\left(M_{x}\right)$ & $* *$ & 1.92 & 1.1625 & 1.05 & & $* *$ & 2.33 \\
$\left(B_{I}\right)$ & $* *$ & 2.25 & 1.2208 & 1.08 & & $* *$ & 2.56 \\
$\left(M_{D}\right)$ & $* *$ & 1.52 & 1.02 & 1.01 & & $* *$ & 2.05 \\
$\left(B_{I I}\right)$ & $* *$ & 1.79 & 1.15 & 1.0397 & & $* *$ & 2.21 \\
$(v)$ & 1.14 & 1.14 & 1.17 & 1.18 & & 1.78 & 1.78 \\
\hline
\end{tabular}

**Not applicable due to symmetric nature of the load.

Table 6

Effect of vehicle speed on impact factor

\begin{tabular}{ccccc}
\hline \multicolumn{5}{c}{ Vehicle speed $(\nu) \mathrm{m} / \mathrm{sec}$} \\
\hline Response parameter & 5 & 10 & \multicolumn{1}{c}{15} & \multicolumn{1}{c}{20} \\
\hline$\left(M_{z}\right)$ & 1.05 & 1.06 & 1.07 & 1.08 \\
$\left(Q_{Y}\right)$ & 1.04 & 1.06 & 1.0712 & 1.08 \\
$\left(M_{x}\right)$ & 1.16 & 1.24 & 1.52 & 1.92 \\
$\left(B_{I}\right)$ & 1.27 & 1.47 & 1.80 & 2.25 \\
$\left(M_{D}\right)$ & 1.012 & 1.16 & 1.21 & 1.52 \\
$\left(B_{I I}\right)$ & 1.04 & 1.17 & 1.43 & 1.79 \\
$(v)$ & 1.11 & 1.12 & 1.13 & 1.14 \\
\hline
\end{tabular}

Table 7

Effect of span of box-girder bridge on impact factor

\begin{tabular}{clll}
\hline \multicolumn{4}{c}{ Span of box-girder $(L) \mathbf{m}$} \\
\hline Response parameter & 30 & \multicolumn{1}{c}{60} & \multicolumn{1}{c}{90} \\
\hline$\left(M_{z}\right)$ & 1.08 & 1.041 & 1.025 \\
$\left(Q_{Y}\right)$ & 1.08 & 1.06 & 1.03 \\
$\left(M_{x}\right)$ & 1.92 & 1.781 & 1.529 \\
$\left(B_{I}\right)$ & 2.25 & 2.01 & 1.891 \\
$\left(M_{D}\right)$ & 1.52 & 1.218 & 1.010 \\
$\left(B_{I I}\right)$ & 1.79 & 1.44 & 1.29 \\
$(v)$ & 1.14 & 1.125 & 1.103 \\
\hline
\end{tabular}

Table 8

Effect of radius of box-girder bridge on impact factor

\begin{tabular}{ccccc}
\hline \multicolumn{5}{c}{ Radius of box-girder $(R) \mathbf{m}$} \\
\hline Response parameter & 100 & 150 & 250 & $\infty$ \\
\hline$\left(M_{z}\right)$ & 1.0907 & 1.08 & 1.0785 & 1.07 \\
$\left(Q_{Y}\right)$ & 1.092 & 1.081 & 1.0712 & 1.06 \\
$\left(M_{x}\right)$ & 2.5061 & 1.92 & 1.5176 & 1.02 \\
$\left(B_{I}\right)$ & 3.1636 & 2.25 & 1.6766 & 1.21 \\
$\left(M_{D}\right)$ & 2.4597 & 1.52 & 1.3734 & 1.0051 \\
$\left(B_{I I}\right)$ & 2.3198 & 1.79 & 1.50 & 1.14 \\
$(v)$ & 1.1647 & 1.14 & 1.13 & 1.12 \\
\hline
\end{tabular}

increase of radius of curvature.

(vi) Effect of bridge deck surface condition

The surface characteristics play an important role on the dynamic excitation transmitted on the bridge by the moving vehicle. Five categories of surface characteristics have been considered to examine their effect on the response based on the spectral roughness coefficient and classified as very good, good, average, poor and very poor. The effect of any discrete form of roughness such as bump at the approach, construction joints etc are not taken into account. The impact factor corresponding to different response parameters for different categories of 
Table 9

Effect of random road surface condition on impact factor

\begin{tabular}{cccccc}
\hline \multicolumn{7}{c}{ Random road surface condition } \\
\hline Response parameter & Very good & Good & Average & Poor & Very poor \\
\hline$\left(M_{z}\right)$ & 1.06 & 1.08 & 1.33 & 1.72 & 2.00 \\
$\left(Q_{Y}\right)$ & 1.07 & 1.08 & 1.26 & 1.60 & 1.83 \\
$\left(M_{x}\right)$ & 1.91 & 1.92 & 1.93 & 2.00 & 2.05 \\
$\left(B_{I}\right)$ & 2.24 & 2.25 & 2.26 & 2.28 & 2.29 \\
$\left(M_{D}\right)$ & 1.51 & 1.52 & 1.53 & 1.55 & 1.56 \\
$\left(B_{I I}\right)$ & 1.78 & 1.79 & 1.80 & 1.81 & 1.83 \\
$(v)$ & 1.08 & 1.14 & 1.48 & 1.91 & 2.21 \\
\hline
\end{tabular}

Table 10

Effect of sprung mass $\left(m_{s}\right)$ on impact factor

\begin{tabular}{clllll}
\hline \multicolumn{7}{c}{ Sprung mass $\left(m_{s}\right)$} \\
\hline Response parameter & $0.7\left(m_{s}\right)$ & $0.9\left(m_{s}\right)$ & $1\left(m_{s}\right)$ & $1.1\left(m_{s}\right)$ & $1.3\left(m_{s}\right)$ \\
\hline$\left(M_{z}\right)$ & 1.1586 & 1.10 & 1.08 & 1.0782 & 1.06 \\
$\left(Q_{Y}\right)$ & 1.12 & 1.09 & 1.08 & 1.07 & 1.05 \\
$\left(M_{x}\right)$ & 1.9189 & 1.92 & 1.9196 & 1.92 & 1.917 \\
$\left(B_{I}\right)$ & 2.2453 & 2.24 & 2.2469 & 2.24 & 2.2411 \\
$\left(M_{D}\right)$ & 1.5154 & 1.52 & 1.5165 & 1.52 & 1.5188 \\
$\left(B_{I I}\right)$ & 1.7771 & 1.78 & 1.79 & 1.7858 & 1.7882 \\
$(v)$ & 1.2880 & 1.19 & 1.14 & 1.13 & 1.1223 \\
\hline
\end{tabular}

Table 11

Effect of vehicle suspension stiffness $\left(k_{s}\right)$ on impact factor

\begin{tabular}{cccccc}
\hline \multicolumn{5}{c}{ Vehicle suspension stiffness $\left(k_{s}\right)$} \\
\hline $\begin{array}{c}\text { Response } \\
\text { parameter }\end{array}$ & $0.7\left(k_{s}\right)$ & $0.9\left(k_{s}\right)$ & $1\left(k_{s}\right)$ & $1.1\left(k_{s}\right)$ & $1.3\left(k_{s}\right)$ \\
\hline$\left(M_{z}\right)$ & 1.12 & 1.11 & 1.08 & 1.07 & 1.06 \\
$\left(Q_{Y}\right)$ & 1.10 & 1.09 & 1.08 & 1.07 & 1.06 \\
$\left(M_{x}\right)$ & 1.92 & 1.92 & 1.92 & 1.92 & 1.92 \\
$\left(B_{I}\right)$ & 2.24 & 2.24 & 2.24 & 2.24 & 2.24 \\
$\left(M_{D}\right)$ & 1.52 & 1.52 & 1.52 & 1.52 & 1.52 \\
$\left(B_{I I}\right)$ & 1.78 & 1.78 & 1.78 & 1.78 & 1.78 \\
$(v)$ & 1.18 & 1.17 & 1.14 & 1.13 & 1.12 \\
\hline
\end{tabular}

road surface has been presented in Table 9. It can be observed that surface roughness has more significant effect on impact factor due to vertical deflection, bending moment and shear force than any other parameters considered in the study. However, the effect of surface roughness on torsion, distortion and their corresponding bi-moments are comparatively less significant. Thus, surface roughness is one of the predominant factors, which may cause large increase in the response magnitude. Increased deck roughness due to poor maintenance may cause appreciable increase in the impact factor in all span range.

(vii) Effect of sprung mass and suspension stiffness of vehicle

It can be observed from the Table 10 that the impact factors of most responses decrease with the increase of sprung mass. This is due to increase of static response of the bridge. However, the impact factors of most responses are unaffected with the increase of suspension stiffness of vehicle as can be observed from Table 11.

(ix) Effect of acceleration of vehicle

Table 12 shows the effect of vehicular acceleration (constant) on the impact factors. Dynamic responses have been evaluated with the initial velocity of $5 \mathrm{~m} / \mathrm{sec}$ and with different constant values of vehicular acceleration. It has been observed that while the impact factors corresponding to bending moment, shear force and vertical deflection have increased, the impact factors corresponding to torsional and distortional moments, their respective bi-moments have reduced significantly with the increase of acceleration of vehicular motion. 
Table 12

Effect of acceleration of vehicle on impact factor

\begin{tabular}{ccccc}
\hline \multicolumn{5}{c}{ acceleration $(a) \mathrm{m} / \mathrm{sec}^{2}$} \\
\hline Response parameter & 10 & 5 & 2.5 & 0 \\
\hline$\left(M_{z}\right)$ & 1.31 & 1.25 & 1.20 & 1.08 \\
$\left(Q_{Y}\right)$ & 1.24 & 1.22 & 1.19 & 1.08 \\
$\left(M_{x}\right)$ & 1.16 & 1.22 & 1.31 & 1.92 \\
$\left(B_{I}\right)$ & 1.27 & 1.35 & 1.47 & 2.25 \\
$\left(M_{D}\right)$ & 1.02 & 1.14 & 1.20 & 1.52 \\
$\left(B_{I I}\right)$ & 1.13 & 1.19 & 1.28 & 1.79 \\
$(v)$ & 1.38 & 1.31 & 1.27 & 1.14 \\
\hline
\end{tabular}

\section{Conclusions}

The dynamic response of thin-curved box girder bridge due to moving vehicle has been studied considering surface unevenness of the deck as the random input to the vehicle wheels. Impact factors for several response parameters have been presented for the variations of several bridge and vehicle parameters. A computationally less expensive and realistic three noded thin-walled box beam element has been utilized for the modeling of the bridge. The applicability of such an element for the dynamic analysis has been verified by evaluating the modal parameters of a curved box girder model theoretically as well as experimentally. The element has performed highly satisfactorily for the evaluation of dynamic response of coupled bridge-vehicle system, since most of the observations made are supportive of the earlier works [6,7]. However, the following important conclusions may be made from the present study in respect of evaluation of impact factor due to vehicle-induced vibration on a thin walled box girder bridge.

- Due to the effect of centrifugal force in a curved bridge, the impact factors due to torque, distortion and their corresponding bi-moments are generally higher than those of vertical deflection, bending moment and shear force. It has been observed that the impact factors of torque, distortion and their corresponding bi-moments rapidly increase with vehicle speed, while those of vertical deflection, bending moment and shear force do not show such tendency.

- Surface roughness is one of the predominant factors, which may cause large increase in the magnitude of dynamic response particularly of vertical deflection, bending moment and shear force.

- The transverse load position on the bridge deck as well as number of vehicles at a section influence the values of impact factor.

- Vehicle suspension stiffness has lesser influence on impact factors corresponding to different response parameters in comparison to the sprung mass variation.

- It has been observed that by completely neglecting the effect of damping will overestimate the impact factors of vertical deflection, bending moment and shear force for a curved box-girder bridge.

\section{References}

[1] B.I. Maisel, Analysis of concrete box beams using small computer capacity, Canadian Journal of Civil Engineering 12 (1985), $265-278$.

[2] B. Kermani and P. Waldron, Analysis of continuous box girder bridges including the effects of distortion, Computers and Structures $\mathbf{4 7}$ (1993), 427-440.

[3] S.H. Zhang and L.P.R. Lyons, A Thin-Walled Box Beam Finite Element for Curved Bridge Analysis, Computers and Structures 18 (1984), 1035-1046.

[4] A.G. Razaqpur and H.G. Li, A Finite Element with Exact Shape Functions for Shear Lag Multi-cell Box Girders, Computers and Structures 39 (1991), 155-163.

[5] D.R. Schelling, N.H. Galdos and M.A. Shahin, Evaluation of impact factors for horizontally Curved steel Box-girder Bridges, Journal of Structural Engineering ASCE 118 (1992), 3203-3221.

[6] D.Z. Huang, T.L. Wang and M. Shahaway, Vibration of horizontally curved Box-girder bridges due to vehicles, Journal of computers and structures 68 (1998), 513-528.

[7] D.Z. Huang, Dynamic analysis of steel curved box girder bridges, Journal of Bridge Engineering ASCE 6 (2001), 506-513.

[8] Y.B. Yang, C.-M. Wu and J.-D. Yau, Dynamic response of a horizontally curved beam subjected to vertical and horizontal moving loads, Journal of Sound and Vibration 242 (2001), 519-537.

[9] M.F. Green and D. Cebon, Dynamic interaction between heave vehicles and highway bridges, Journal of computers and structures $\mathbf{6 2}$ (1997), 253-264. 
[10] Y.B. Yang and Y.S. Wu, A versatile element for analyzing vehicle-bridge interaction response, Engineering structures 23 (2001), $452-469$.

[11] D. Yadav and H.C. Upadhyay, Heave-Pitch-Roll Dynamics of vehicle with a variable velocity over a non-homogeneously profile flexible track, Journal of Sound and Vibration 164 (1993), 337-348.

[12] D.Z. Huang, T.L. Wang and M. Shahaway, Dynamic behavior of horizontally curved I- girder bridges, Journal of computers and structures 57 (1995), 703-714.

[13] H. Honda, Y. Kajkawa and T. Kobori, Spectra of road surfaces on bridges, Journal of Structural Engineering ASCE 108 (1982), $1956-1966$.

[14] M. Shinozuka, Simulation of multivariate and multidimensional random processes, The Journal of the Acoustical Society of America 49 (1971), 357-367.

[15] F.T.K. Au, Y.S. Cheng and Y.K. Cheung, Effects of random road surface roughness and long-term deflection of prestressed concrete girder and cable-stayed bridges on impact due to moving vehicles, Computers and Structures 79 (2000), 853-872.

[16] D.R.J. Owen and E. Hinton, Finite Elements in Plasticity: Theory and Practice, Pineridge Press Limited, Swansea, U.K. 1980.

[17] S.H. Zhang, The finite element analysis of thin-walled box spine-beam bridges, Ph.D. Thesis, The City University, London.1983.

[18] J. Inman Deniel, Engineering Vibration, Prentice Hall, Inc., second edition.2001.

[19] K. Henchi, M. Fafard, G. Dhatta and M. Talbot, An efficient Algorithm for Dynamic analysis of bridge under moving vehicle using a coupled model and physical components approach, Journal of Sound and Vibration 12 (1998), 663-683. 

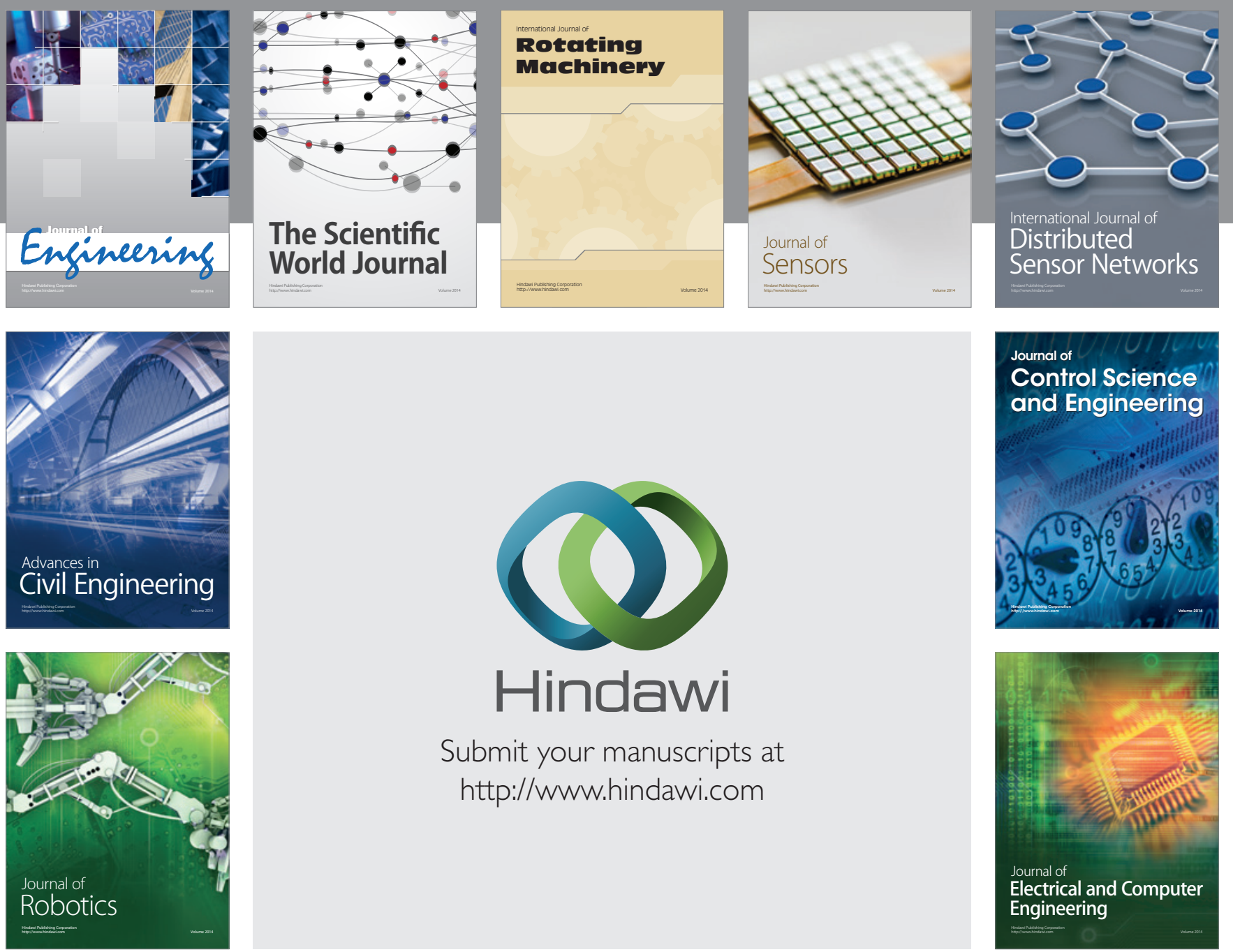

Submit your manuscripts at

http://www.hindawi.com
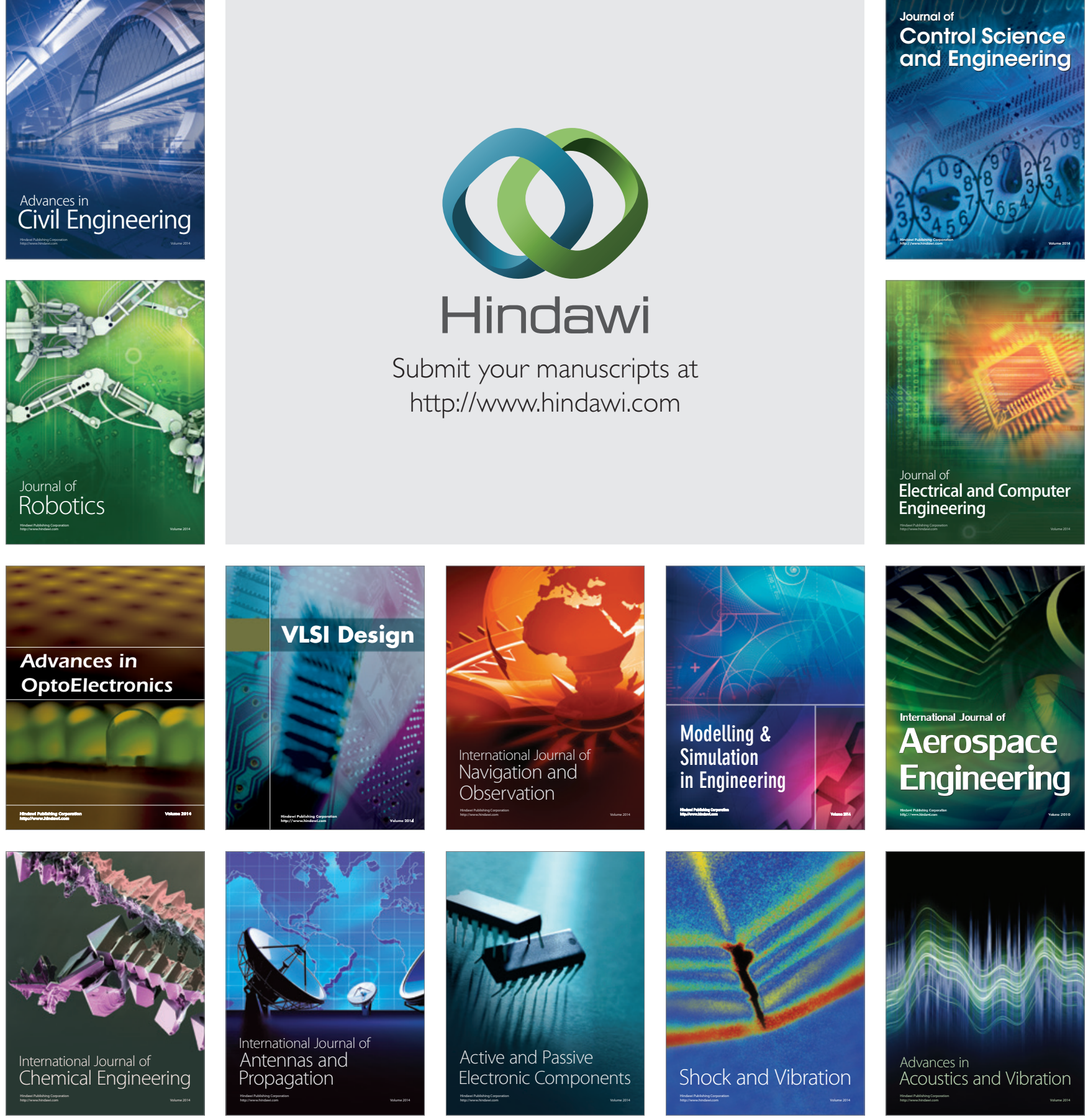